
$\left(\mathrm{XV}^{\mathrm{e}}-\mathrm{XIX}{ }^{\mathrm{e}}\right.$ siècle)

\title{
Un discours de légitimation du géographe et de la géographie par la renommée et la célébrité : l'exemple de Jean-Baptiste d'Anville (1697-1782)
}

\section{Lucile Haguet}

\section{OpenEdition}

\section{Journals}

Édition électronique

URL : https://journals.openedition.org/geohist/1027

DOI : 10.4000/geohist.1027

ISSN : 2264-2617

Éditeur

Association française de la Revue de géographie historique

\section{Référence électronique}

Lucile Haguet, « Un discours de légitimation du géographe et de la géographie par la renommée et la célébrité : l'exemple de Jean-Baptiste d'Anville (1697-1782)», Revue de géographie historique [En ligne] 17-18 | 2020, mis en ligne le 05 mai 2021, consulté le 12 juin 2021. URL : http:// journals.openedition.org/geohist/1027 ; DOI : https://doi.org/10.4000/geohist.1027

Ce document a été généré automatiquement le 12 juin 2021

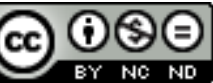

$\mathrm{Ce}(\mathrm{tte})$ œuvre est mise à disposition selon les termes de la Licence Creative Commons Attribution Pas d'Utilisation Commerciale - Pas de Modification 4.0 International. 


\title{
Un discours de légitimation du géographe et de la géographie par la renommée et la célébrité : l'exemple de Jean-Baptiste d'Anville (1697-1782)
}

\author{
Lucile Haguet
}

1 Pour amorcer sa carrière, un aspirant géographe du siècle des Lumières devait surmonter de nombreux obstacles. Sans une reconnaissance minimale de ses talents, il ne peut produire de la connaissance, car c'est grâce à elle qu'il obtient les moyens de construire son œuvre : sources d'information, financement de l'édition de ses travaux, temps libre. Mais naturellement, sans œuvre produite, il ne saurait y avoir de reconnaissance. Tout début de carrière était donc une quadrature du cercle, période hasardeuse et cruciale. Le jeune savant dispose de peu de moyens financiers à moins de bénéficier d'un solide soutien familial. Il n'a que peu de temps à consacrer à son art, car il lui faut gagner sa vie. Il jouit de peu de reconnaissance, ce qui limite son accès à l'information, faute d'avoir encore beaucoup accompli. Son patrimoine économique et social est à construire, sauf à avoir hérité ou contracté un mariage avantageux. Pour bâtir sa carrière, le jeune savant ne peut compter que sur sa ténacité et cultiver ses premiers soutiens. Mais vers qui se tourner? Qui fait un savant au XVIII siècle? Les carrières ne se font plus uniquement à la cour et pour amorcer un début de légitimité, il convient de se faire connaître de la République des lettres et du public. Afin de se consacrer à ses recherches plutôt qu'à l'enseignement, le soutien financier d'un mécène est indispensable. Une réputation, même balbutiante, est indispensable pour se lier à ses premiers protecteurs, être identifié par ses premiers commanditaires, et obtenir peu à peu les moyens de ses ambitions.

2 Jean-Baptiste d'Anville (1697-1782), géographe majeur du XVIII siècle, n'est ni un héritier ni un coureur de dot (Fig. 1). Fils d'un maitre tailleur d'habit, non dépourvu de 
moyens mais sans véritablement de fortune, sans appuis familiaux dans le milieu de la cartographie, il doit se construire seul. Très rapidement, il prend conscience de la nécessité de se faire connaître et saisit les moyens de l'époque, comme les journaux qui prennent leur essor (Verdier, 2014). Il comprend que son nom doit être gage de qualité, afin que le public puisse reconnaître la supériorité de ses cartes et les préférer à celles de ses concurrents. Pour y parvenir, d'Anville use de nombreux leviers. Il fait jouer son cercle de sociabilité et s'insère autant que possible dans les institutions existantes, françaises ou étrangères. Nouveauté, il rend public les raisonnements qui ont conduit à la construction de ses cartes. Pour asseoir sa crédibilité, il se construit un personnage, celui du savant solitaire, exclusivement dédié à son travail.

Cette renommée perdure après sa mort et prend une nouvelle dimension : de grand géographe, il passe "grand homme ». Après avoir été qualifié de "fameux », voire de " célèbre » de son vivant, il est choisi après sa mort pour apparaitre dans la "galerie métallique des grands hommes", une série de médailles frappées à l'effigie des hommes et des femmes qui font la fierté de la France entre 1816 et 1826. On peut s'interroger sur la nature de cette reconnaissance. Est-ce une exagération ou d'Anville a-t-il véritablement bénéficié d'une renommée exceptionnelle? Si c'est le cas, quelle a été l'incidence de cette reconnaissance du "public » sur son parcours? Lui a-t-elle permis de compenser une reconnaissance tardive par les académies et de lancer sa carrière ? Et après sa mort, la renommée du géographe a-t-elle découlé de celle acquise de son vivant, ou bien est-ce une construction élaborée par les géographes du XIX ${ }^{\mathrm{e}}$ siècle pour affermir la légitimité d'une discipline qui peine à s'imposer dans les institutions? L'étude attentive de la réception des œuvres du savant, ainsi que celle du savant lui-même, de son vivant, puis après sa mort, aux XVIII ${ }^{e}$ et $\mathrm{XIX}^{\mathrm{e}}$ siècles, devrait apporter quelques réponses. 
Fig. 1: Benjamin Duvivier, Augustin de Saint-Aubin, J. B. Bourguignon d'Anville, après 1782.

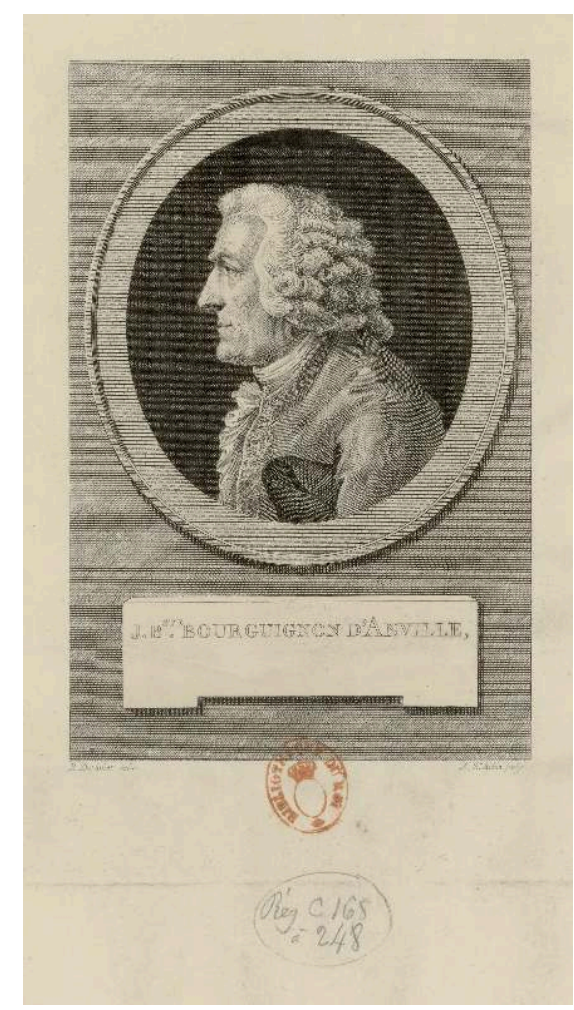

\section{Peut-on parler de gloire, célébrité, renommée à propos de d'Anville?}

Le "célèbre d'Anville", pour reprendre les termes de ses contemporains, n'est plus guère connu aujourd'hui que de quelques historiens, car sa renommée s'éteint au début $\mathrm{du} \mathrm{XX}^{\mathrm{e}}$ siècle. C'est pourquoi le présenter est indispensable. Né en 1697 à Paris, JeanBaptiste d'Anville est un géographe érudit, autrement dit un "géographe de cabinet ", expression à l'origine péjorative, plutôt employée par les géographes explorateurs, pour discréditer les savants qui effectuaient sans voyager le nécessaire travail de compilation des sources (Haguet, Hofmann, 2017).

D'Anville n'est pas un géographe de terrain. De sa vie, il ne dépassera jamais Alençon, où son gendre possède un château. Il dessine le monde depuis sa table de travail, à partir des matériaux issus de sa bibliothèque. Il réalise une multitude de notes et de fiches, selon une méthode proche de celle de Guillaume Delisle. Selon toute vraisemblance, d'Anville a bénéficié des conseils de son aîné. Les deux hommes enseignaient la géographie au jeune Louis XV et étaient proches de l'érudit Louis Dufour de Longuerue (Mormiche, 2017). En 1719, alors que d'Anville n'a que 22 ans, Delisle lui fait même don d'une carte pour améliorer ses propres travaux, ainsi qu'on en use avec un jeune protégé prometteur (d'Anville, 1719, p. 77).

L'élève surpasse son maître. Certes, il bénéficie de nouvelles sources. Mais il est aussi plus exhaustif et méticuleux. Sa supériorité tient surtout à un outil qu'il s'est construit : le Traité des mesures itinéraires publié en 1769. Ce traité va lui permettre d'utiliser et de 
mettre en concordance toutes les sources qu'il récolte, qu'elles soient antiques, étrangères ou simplement provinciales. Rappelons qu'il n'existe pas, avant la première définition du mètre le 26 mars 1791 par l'Académie des sciences, de mesure universelle, à l'exception du pied imposé par l'Empire romain. Selon les lieux et les temps, les unités de mesure diffèrent, alors même qu'elles portent parfois le même nom. En retrouvant la valeur de chacune par un patient travail d'analyse textuelle, d'Anville peut exploiter pleinement toutes les sources dont il dispose. Par ailleurs, il se distingue par ses talents de dessinateur, développés dans l'enfance ${ }^{1}$ qui lui permettent de réaliser des cartes très lisibles.

7 À 21 ans, il participe à l'éducation du jeune Louis XV, reçoit quelques mois plus tard un brevet de géographe ordinaire du roi et est remarqué par le Régent. C'est le début d'une carrière de protégé des ducs d'Orléans. Dès 23 ans, il travaille pour le roi du Portugal, Jean V (Mandroux-França, 1986; Furtado, 2012). La trentaine à peine dépassée , il obtient ses premières commandes pour illustrer des récits de voyages. Si les débuts sont prometteurs, la reconnaissance académique, elle, tarde. Il ne devient membre de l'Académie des sciences de Saint-Pétersbourg qu'en 1748 vers 51 ans, entre à l'Académie royale des inscriptions et belles-lettres en 1754 vers 57 ans, puis à l'Académie royale des sciences en 1775. Il a alors 77 ou 78 ans. Par comparaison, Condorcet y est admis à 25 ans. C'est bien la preuve que la géographie peine à exister dans les institutions : cette tardive reconnaissance contraste avec l'audience française et internationale de ses travaux.

D'Anville présenté, reste à savoir de quoi la réputation, la gloire, et la célébrité, déclinaisons de la renommée, sont le nom. Dans l'ouvrage Figures publiques, l'invention de la célébrité (1750-1850) (Lilti, 2014, 84-91), Antoine Lilti propose la typologie suivante. La "gloire », héritière de la figure du héros, n'existe que post-mortem et appartient à la mémoire collective. La « réputation » se définirait simplement par la reconnaissance des pairs, sans s'étendre au-delà de ce cercle. La « célébrité » se différencie de la gloire et de la réputation en ce qu'elle n'est pas forcément posthume et n'est pas unanime (Lilti, 2014, 12-13). Elle se distingue de la "réputation » dans la mesure où la raison même qui a permis à la personne de devenir célèbre, talent, courage ou même crime, devient secondaire (Lilti, 2014, 14). Par ailleurs, la célébrité est intrinsèquement liée à l'image et sont incontestablement célèbres les personnes dont on vend pour quelques sous les portraits gravés.

9 Cependant, l'ensemble du champ lexical de la renommée ne fait pas l'objet de définitions par l'historien, comme la "notoriété » qui paraît ainsi être utilisée comme un terme générique qui englobe toutes les formes de reconnaissance ou la " renommée ». ${ }^{2}$ Nous userons à notre tour de ces termes dans cette acception générale.

Rappelons, si cela était nécessaire, que c'est Lilti qui construit ces définitions. Elles ne préexistent pas telles quelles au XVIII ${ }^{\mathrm{e}}$ et $\mathrm{XIX}^{\mathrm{e}}$ siècle, où leurs contemporains peinent à appréhender des phénomènes qui parfois émergent tout juste. Ceux-là multiplient à l'envi les adjectifs ( fameux», «illustre») ou les expressions comme «estime publique ». Du reste, plus que des définitions, Lilti propose une typologie de la renommée. Incontestablement, Rousseau et Voltaire sont célèbres. Ils sont connus même par ceux qui ne les ont pas lus et sont reconnus au sens figuré comme au sens propre. Appliquée à notre cas, en l'occurrence d'Anville, cette typologie révèle qu'à l'aune des traces de sa réception, dans les écrits de ses contemporains et successeurs, le 
géographe jouit d'une solide réputation de son vivant et accède même pour quelques décennies à une gloire posthume, voire une forme de célébrité.

11 Les typologies, stimulantes pour les analyses générales, ne fonctionnent que rarement parfaitement pour le cas particulier, ce qu'admet Antoine Lilti dès son introduction, puisqu'il rappelle qu' «il serait absurde de plaider pour une étanchéité absolue entre réputation, célébrité et gloire » (Lilti, 2014, 15).

Jean-Baptiste d'Anville était avant tout connu de son vivant par ses admirateurs et ses clients. Mais quand un cercle de clients et d'admirateurs est très large, et que le nom du savant cartographe devient presque une marque, un peu comme les cartes Michelin, ne touche-t-on pas à une forme de célébrité ? D'Anville, par ailleurs, en possède quelques caractéristiques : par exemple, ses portraits se multiplient après sa mort et leur qualité progressivement décroît au fil des imitations, qui s'éloignent de l'original, un phénomène typique de la célébrité (Lilti, 2014, 78-84). De même, le cas d'Anville participe plus de la célébrité que de la réputation ou de la gloire posthume par l'ambiguïté des réactions qu'il suscite: on lui reproche sa vanité, on l'accuse de ne pas s'y connaître en mathématiques ou encore de mal écrire (Dacier, 1785, 74).

On serait alors tenté de parler dans le cas de d'Anville de grande réputation ou de petite célébrité, mais ce serait réintroduire une continuité entre les deux phénomènes, qui ne se distingueraient que par leur degré d'intensité, continuité que Lilti s'attache justement à remettre en cause pour montrer qu'il s'agit bien de deux phénomènes distincts, même s'ils se superposent parfois.

14 Mais si la réception de d'Anville et de son œuvre paraît si paradoxale, si inclassable, c'est peut-être parce qu'elle est particulièrement fabriquée et instrumentalisée, de son vivant, puis à sa mort. Nous postulons que la renommée d'un géographe devient indispensable à sa carrière au XVIII ${ }^{e}$ siècle, puis nécessaire à la légitimation de la géographie érudite au XIX ${ }^{e}$ siècle. Il devient incontournable qu'un grand nombre de personnes, le "public» comme l'appelle d'Anville, et qui est plus étendu que les simples clients de cartographie ou les spécialistes, parlent de lui, de son vivant, et après sa mort.

Puisqu'il s'agit d'étudier la réception de d'Anville, comme figure savante, tout autant que celle de son œuvre, il ne parait pas inutile d'évoquer les discours tenus sur le géographe, de son vivant et après sa mort, même si, comme on l'a vu, les adjectifs comme «fameux » ou " célèbres » n'ont pas un sens nettement défini. De son vivant, d'Anville était qualifié de célèbre et fameux avant même ses quarante ans (Castel, 1737, p.1156-1226). Cependant, il ne faut pas s'exagérer la récurrence de ces qualificatifs, qui deviennent au contraire presque systématiques après sa mort. De son vivant, il reste le plus souvent : «M. d'Anville».

Dans l'ensemble, les louanges reçues ne sont pas distinctes de ses travaux, qui sont au contraire évoqués explicitement, soit un discours typique de la réputation. Ses alterego le reconnaissent comme une référence. Le géographe Didier Robert de Vaugondy, dans son Essai sur l'histoire de la géographie de 1755, ne le cite pas moins de 23 fois, en des termes élogieux: "les [cartes les] plus récentes sont celles de M. d'Anville; elles renferment un détail supérieur à tout ce qui a été publié dans ce genre » (Robert de Vaugondy, 1755, 403-404). Chez les utilisateurs de cartes, comme les voyageurs, il est considéré comme une référence. Les explorateurs vantent la précision quasi prédictive 
de ses cartes qui localisent des lieux qui n'ont pas encore été explorés, ainsi que l'observe Bougainville, qui connaissait personnellement d'Anville :

Celle qui m'a donné le plus de lumières, est la carte d'Asie de M. Danville, publiée en 1752. [...] J'ajouterai que la Nouvelle Guinée et les iles des Papous approchent plus de la vraisemblance sur sa carte que sur aucune autre que j'eusse entre les mains (Bougainville, 1772, 342).

Les voyageurs par voie de terre se répandent en semblables éloges, comme le comte de Choiseul-Gouffier, diplomate passionné par l'Antiquité, qui explore en 1776 une partie de la Grèce, de l'Asie mineure et de la Syrie, les cartes de d'Anville à la main.

Nous devons à d'Anville des cartes de la Grèce bien supérieures à toutes celles qui avaient paru avant lui : elles sont incontestablement les plus exactes [...] (ChoiseulGouffier, 1822, 17).

De même, les historiens ou encore ceux qu'on appelait alors les "antiquaires ", des érudits spécialisés dans l'Antiquité, reconnaissent la qualité de son travail. Non sans une certaine emphase, Domenico Silvio Passionei, érudit, collectionneur d'art et d'ouvrages anciens, vice-bibliothécaire de la Vaticane, qualifie d'Anville de «divinité géographique » (Marcel, 1904, p.432).

Cependant, dès son vivant, la réputation de d'Anville s'étend au-delà de son public naturel d'érudits et de professionnels. Didier Robert de Vaugondy écrit dans un texte introductif à son Atlas universel qu'à propos des œuvres de d'Anville «il seroit inutile d'en faire l'énumération puisque le public les connoît». Or c'est bien "public » dans son sens de large lectorat qu'il faut entendre ici, puisqu'un public de non-spécialiste, moins lettré, demande à avoir accès à son travail, notamment un public plus populaire ou encore féminin, qui n'a pas eu accès à l'apprentissage du latin :

$C^{\prime}$ est au regret que lui témoignèrent plusieurs personnes de ne pouvoir consulter ses cartes anciennes, faute d'entendre le latin, que nous devons sa Géographie ancienne abrégée, qu'il donna au public en 1768 (Dacier, 1802, 33)

Sans être célèbre au sens de Lilti, d'Anville jouit donc d'une renommée très étendue.

Cependant, d'Anville ne se contente pas de soigner ses cartes, ses essais et d'une manière générale, son travail. Il soigne aussi son image. Surtout dans la seconde moitié de sa vie, il s'est attelé à construire sa propre légende en se définissant comme un " homme-travail ", ayant renoncé à toute sociabilité, même savante. Il déclare à ses correspondants avoir « fait une sorte de vœu de ne pas sortir le samedi $»^{3}$, insiste sur la longévité de sa carrière (D'Anville, 1777, 109)4, et se présente comme un savant solitaire « qui n'a point formé d'élève » (D'Anville, 1777, 110), faute d'avoir trouvé un individu à sa mesure.

Il n'en reste pas moins que d'Anville est très loin d'une célébrité à la Voltaire. Nul ne se soucie de la vie intime du géographe. D'Anville reste connu avant tout pour ses travaux, et les détails de sa vie domestique n'intéressent pas. Enfin, son portrait n'est pas diffusé de son vivant. Certes, il existe un portrait à l'huile du géographe, sans doute peint par Louis Tocqué dans les années 1750. Il ne fut pas diffusé en gravure. Il n'était sans doute pas accroché chez les d'Anville, au Louvre, car ses filles et ses proches n'en avaient pas connaissance. Louis Charles Joseph de Manne, fils de Nicolas Joseph, secrétaire de d'Anville, était si persuadé que personne n'avait fait le portrait du géographe de son vivant, qu'il fit réaliser un portrait posthume du géographe (De Manne, 1834, XXVj, $\mathrm{n}^{\circ} 1$ ). Le portrait $\mathrm{n}^{\prime} \mathrm{a}$ donc pas été commandé par d'Anville et était apparemment conservé par une autre personne dont l'identité reste inconnue. Le tableau fut ensuite 
acquis par Antoine Letronne, admirateur de d'Anville, avant d'entrer dans les collections d'État en 1849. Il est aujourd'hui conservé à Versailles. ${ }^{5}$

Le visage et les mœurs de d'Anville ne faisaient donc pas l'objet, de son vivant, d'un intérêt particulier. En revanche, par la renommée de son travail et l'image qu'il se construit lui-même d'ermite de la cartographie, le géographe remplit certaines conditions nécessaires, bien que non suffisantes, pour accéder à la gloire posthume.

De fait, les géographes, et surtout les géographes de cabinet atteignent rarement la gloire et ne sont guère érigés en "grands hommes", c'est-à-dire en figures patriotiques (Bonnet, 2001). Cela tient, d'abord, à ce qu'au XVIII esiècle, peu de savants se définissent comme géographes ou sont identifiés comme géographes par leurs contemporains, bien que la géographie érudite ait appartenu à leur champ de recherches. En 1855, Louis Vivien de Saint-Martin, rédacteur des Nouvelles annales de la géographie, prononce un discours devant la Société de géographie, dont il est l'exsecrétaire général. Publié sous le titre De l'État de la cartographie en Europe, ce discours est destiné à dénoncer la décadence de la géographie en France, et notamment la géographie de cabinet. Par contraste, Vivien de Saint-Martin évoque la faveur dont elle jouissait, selon lui, au XVIII ${ }^{\mathrm{e}}$ siècle :

La géographie savante était alors en grand honneur au sein de l'Académie des inscriptions, où les fréquentes lectures de de la Nauze, de Bougainville, de Gibert, de de la Barre, de Bonamy, et surtout celles du profond et du judicieux Fréret, captivaient l'attention et devenaient l'objet de fructueuses controverses. D'Anville s'était nourri de ces fortes études (Vivien de Saint-Martin, 1855, 13).

Or parmi les noms cités par Vivien de Saint-Martin, aucun d'entre eux n'est véritablement connu comme géographe. Selon leurs éloges académiques, Louis Jouard de La Nauze est un jésuite historien de l'Antiquité, Bougainville, un explorateur, Joseph Balthazar Gibert, un philologue et historien, Louis François Joseph de la Barre, historien, Pierre-Nicolas Bonamy, un historiographe et un bibliothécaire, Nicolas Fréret, un historien et linguiste. D'Anville, le géographe érudit, constitue donc une exception.

$\mathrm{Au} \mathrm{XIX}^{\mathrm{e}}$ siècle, les honneurs nationaux ignorent presque totalement les géographes, à l'exception de ces géographes de terrain que sont les explorateurs. Au Panthéon sont inhumés des hommes d'État, des scientifiques, des écrivains, des voyageurs, mais aucun géographe. Peu d'hommages leur sont rendus lors des commémorations nationales qui honorent les grandes figures de la France. Par exemple, parmi les seize statues provisoires d'hommes illustres érigés sur le Pont de la Concorde pour la fête du 4 mai 1851, laquelle commémore le troisième anniversaire de la proclamation de la République par l'Assemblée constituante, ne se trouve aucun géographe (Agulhon, 1998). Les géographes ne font pas l'objet non plus de biographies monographiques au $\mathrm{XIX}^{e}$ siècle. Le Catalogue de l'Histoire de France du département des imprimés de la Bibliothèque n'en fait état d'aucune. On y trouve des personnalités militaires et politiques (Jeanne d'Arc, Colbert), des hommes et femmes de lettres (Fénelon, Madame de Sévigné, Racine), des naturalistes et médecins (Buffon, Pasteur), voire des explorateurs comme La Pérouse, mais jamais de géographes (Bibliothèque nationale, 1855-1895).

Du côté des ouvrages pour la jeunesse, comme le Tour de la France par deux enfants, qui forge l'imaginaire collectif de générations d'écoliers de 1877 au début du XXe siècle, on trouve des militaires, des philosophes, des politiques, des écrivains, des savants 
(Buffon, Jussieu, Lavoisier, Monge, Niepce, Pasteur), des explorateurs (encore La Pérouse), mais toujours aucun géographe (Bruno, Belin, 1877 ; Amalvi, 1998).

D'Anville n'entre donc pas au Panthéon et les historiens ne lui consacrent pas de biographie. Pourtant, il connaît bel et bien une forme de gloire posthume. Certains géographes, comme Conrad Malte-Brun, lui dédient des poèmes d'éloge :

Sans sortir de Paris, mesurant l'univers,

Un mortel a régné sur la terre et les mers.

Le Gange et l'Amazone et Memphis et Byzance,

Tout a subi les loix que lui dictoit sa science.

Ce mortel, c'est D'ANVILLE, et du Strabon français,

L'art offre au Monde ici les purs et nobles traits (Malte-Brun, 1810, 356).

Ici, d'Anville ne devient rien de moins que le nouveau fondateur de la géographie («Strabon français »). Mieux, il surplombe, tel un être suprême, la Terre entière depuis l'Asie jusqu'au Nouveau monde, dont il distingue les contours au passé comme au présent. Comme toute bonne allégorie, il possède un trait distinctif, un attribut presque magique, qui identifie sa spécificité : il est celui qui a mesuré l'univers « sans sortir de Paris ». L'hydrographe Ernest Mouchez, quant à lui, baptise un golfe de Tartarie du nom de d'Anville, golfe dont les détails topographiques portent le nom de grands savants («île Cassini », "presqu'île Cuvier», " pointe Malte-Brun»), signifiant métaphoriquement que d'Anville est le prince d'entre eux. ${ }^{6}$

Comme de son vivant, la renommée du géographe dépasse l'horizon de ses pairs. Des explorateurs, comme Frédéric Cailliaud, lui rendent hommage en gravant son nom sur des pyramides, consacrant ainsi dans la pierre sa position surplombante sur le monde, caractéristique du géographe (Dewachter, 1994, 35-43). Il est aussi connu d'un public qui n'est pas forcément familier de ses œuvres. C'est ainsi que son nom peut être cité dans un roman, sans plus d'explications, comme s'il allait de soi pour le lectorat : en 1846, Honoré de Balzac, au détour d'une phrase de La Cousine Bette, évoque d'Anville « créateur de l'ancien Orient » (Balzac, 1891, 501), sans juger nécessaire de donner plus de précisions. 
Fig. 2: Un exemple de variante déformée du portrait de d'Anville de Benjamin Duvivier, Augustin de Saint-Aubin. Dans Malte-Brun C., 1810, "Sur l'édition complète des Ouvrages de M. d'Anville», Annales des voyages de la géographie et de l'histoire, Paris, Buisson, t. 10, p. 356.

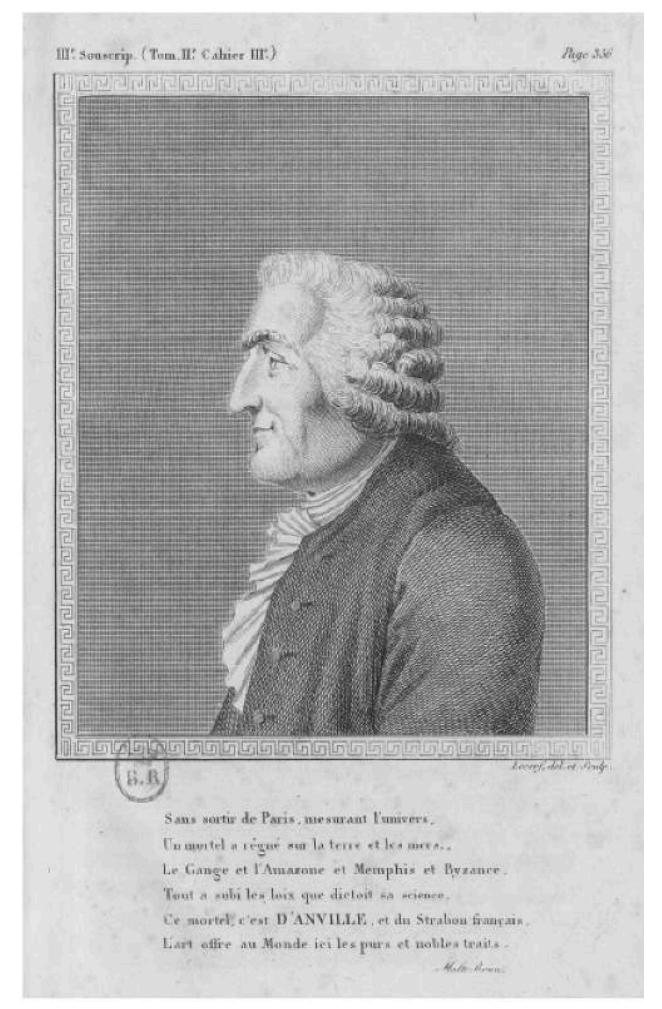

Bibliothèque nationale de France, PHS, G 18346. maintient, voire s'accroît. Son visage, inconnu de son vivant ${ }^{7}$, est diffusé à sa mort par
la gravure, et se déforme, de copies en copies, phénomène que le portrait de d'Anville a en commun avec ceux des célébrités (Lilti, 2014, 84-91) (Fig. 2). ${ }^{8}$ Son visage devient familier au public, grâce à un portrait destiné à la réédition de ses travaux, les đuvres de M. d'Anville, dont le premier tome fut publié en 1835. Le dessin fut commandé à Benjamin Duvivier (1730-1819), et gravé par Augustin de Saint-Aubin (1736-1807). Duvivier et Saint-Aubin étaient spécialisés dans le portrait de personnalités, notamment sous forme de médailles. ${ }^{9}$ Mais ce n'est peut-être pas l'unique raison pour laquelle de Manne fait appel à Duvivier. Ce portrait est en effet réalisé dans des circonstances particulières, puisqu'il est postérieur au décès du géographe. De Manne pensait, rappelons-le, qu'aucun portrait du géographe n'avait été réalisé de son vivant (De Manne, 1834, XXVj, ${ }^{\circ} 1$ ). Faute de mieux, il fit poser Marie-Thérèse, fille de d'Anville, qui lui ressemblait beaucoup. Le procédé surprend aujourd'hui, mais à l'époque, il n'était pas inhabituel de faire poser un autre, soit que le modèle ait disparu, soit qu'il soit trop occupé pour sacrifier à de longues séances de pose. Turgot remplace ainsi le comte d'Angiviller pour Houdon (Allard, Scherf, 2006, 39). David fait poser son fils à la place du premier consul pour son Bonaparte traversant les Alpes (Lentz, 2013, ch. 93). Exemple bien postérieur, mais bien connu, Rodin fait poser Estager, cocher de Tours, pour retrouver les traits de Balzac (Le Normand-Romain, 2007, 165).

Pour assurer au portrait toute la ressemblance requise, on peut imaginer que de Manne ait fait appel à un artiste ayant connu d'Anville. Or, il se trouve que Duvivier a passé 
toute son enfance aux Galeries du Louvre, où son père, graveur également, était logé. Il serait bien étonnant qu'il n'y ait pas croisé d'Anville (Quatremère de Quincy, 1834, 176-191).

Post-mortem, D'Anville tend donc à basculer dans la célébrité. Son nom est plus connu que son œuvre, qui ne suscite pas un vrai intérêt. Dès 1810, Conrad Malte-Brun en fait le constat, qu'il attribue à une certaine frivolité de ses concitoyens :

Or, croirait-on que la collection complète des œuvres de M. d'Anville, du plus célèbre géographe dont se vante la France, collection de morceaux devenus trèsrares et qu'on cherche à haut prix, n'ai trouvé dans la propre patrie de l'auteur, que cinquante-six souscripteurs (Malte-Brun, 1810, 356).

Il est choisi pour apparaître sur l'une des médailles de la Galerie métallique des Grands hommes. Une statue lui est consacrée sur l'Hôtel de ville de Paris (Hillairet, 1997, 30 et 411) et on baptise une rue à son nom dans le XIV e arrondissement (Gourdon de Genouillac, 1879, t. 1, 390). Pour autant, être statufié sur l'Hôtel de ville n'est pas une garantie de célébrité : on publie bientôt un livret d'explication pour connaître les titres de gloire des parisiens sculptés (Veyrat, 1892). La figure de d'Anville n'en est pas moins présent dans l'espace public.

Une impression de paradoxe se dégage de ce tableau. D'Anville paraît connaître une forme de gloire posthume. Or, de son vivant comme après sa mort, il n'a jamais vraiment fait l'unanimité. De son temps, ses collègues de l'Académie royale des belles lettres lui reprochent de ne pas savoir écrire et ceux de l'Académie royale des sciences de ne pas être mathématicien ou astronome. Après sa mort, les géographes de terrain n'en font pas un des leurs. Quant à la célébrité de d'Anville, elle peut laisser sceptique. La notoriété du géographe ne paraît pas mériter ce qualificatif, quand bien même cet adjectif, au XIX ${ }^{\mathrm{e}}$ siècle, est presque systématiquement accolé à son nom. La réputation, bien réelle de d'Anville, paraît plutôt avoir été astucieusement mise en valeur, de son vivant et après sa mort, dans des buts bien précis.

\section{L'orchestration de la renommée de d'Anville de son vivant, terreau d'une carrière}

Le temps est la principale matière première du géographe érudit, et sans doute la plus précieuse. Avec l'argent, qui permet d'imprimer de coûteuses cartes, le temps est le nerf de la guerre. D'Anville y revient sans cesse. Il lui a fallu 37 ans pour finir sa carte d'Égypte (d'Anville, 1766, IV), il prévoit au moins huit ans pour réaliser les 16 cartes du projet de géographie soumis au duc d'Orléans. ${ }^{10}$ La cartographie érudite se construit dans une temporalité distendue où on ne compte pas en semaines ou en mois, mais en années, voire en décennies. À la fin de sa vie, dans un texte biographique, d'Anville insiste encore une fois sur cette question du temps disponible, qui s'est révélée cruciale dès les premières années de sa carrière : «Un désir très-ardent de n'être point distrait dans les études que je m'étois proposé de faire, m'avoit éloigné d'une occupation qui consiste à donner des leçons [...] $\gg{ }^{11}$ Ainsi, sans beaucoup de temps, il n'est pas de géographie érudite possible. Elle s'avère incompatible avec d'autres activités rémunératrices mais jugées peu stimulantes et chronophages, comme l'enseignement, ou encore avec les intérêts commerciaux, qui encouragent une publication fréquente et régulière de cartes, plutôt qu'un perfectionnisme patient. 
Les exigences de d'Anville pourraient sembler démesurées. Pourtant, aucun de ses successeurs, faute de disposer des mêmes conditions de travail, ne parviendra à l'égaler. Ainsi, Pierre Lapie au XIX ${ }^{e}$ siècle, à défaut de bénéficier de soutien institutionnel, doit publier ses cartes rapidement pour rentabiliser son travail scientifique, au détriment de sa qualité, ainsi que le déplore Vivien de Saint-Martin, qui dénonce la nécessité économique qui encourage à une

production multipliée, au milieu de laquelle il est impossible de réserver à la pensée le temps nécessaire pour la maturité des recherches, aussi bien pour la critique des matériaux et la lente élaboration des éléments accumulés. D'Anville, Messieurs, a consacré quinze années entières à la publication seule de ses grandes cartes générales des parties du monde, qui ne forment que vingt-trois feuilles de moyenne grandeur, et cela avec un travail de treize à quatorze heures chaque jour dont aucune préoccupation étrangère à ses travaux ne le détourna jamais un seul instant (Saint-Martin, 1855, 15-16).

Pour Vivien de Saint-Martin, le temps est clairement une des clefs de la méthode de travail du géographe.

D'Anville ne jouissait pas de rentes suffisantes pour vivre. Même cumulés, la dot de sa femme, son fonds de commerce cartographique, les commandes qui lui sont passées, ne sont pas suffisamment rémunératrices. Le géographe aurait dû se résoudre au préceptorat. Le mécénat des ducs d'Orléans va lui permettre de s'en affranchir, tout en lui permettant de financer la gravure et l'impression de ses cartes avec une qualité optimale. Or, on s'en doute, on ne devient pas, sans quelque réputation, le protégé des ducs d'Orléans.

L'enjeu de la réputation est perçu d'emblée chez d'Anville, qui dès 19 ans, publie une « Description de l'isle de Ceylan » en 1717, dans le Mercure de France. Très tôt, le jeune homme dépourvu d'appuis familiaux obtient d'être régulièrement publié dans le journal qui connaît une des plus larges audiences en Europe et participe aux polémiques de son temps (Verdier, 2017, 237-259). La réussite de ses débuts n'est pas assez solide pour lui permettre d'abandonner rapidement la scène publique que constituent les journaux. Certes d'Anville est, dès ses 22 ans, géographe du roi et enseigne la géographie au jeune Louis XV. Mais le soutien royal est précaire. Les titres sont prestigieux, mais ce n'est encore que le pied à l'étrier. Quand ses services ne sont plus nécessaires à l'éducation du jeune roi, d'Anville perd les pensions afférentes.

41 Pendant plus de 30 ans, le géographe continue à écrire dans le Mercure pour se faire un nom auprès de ses pairs, d'éventuels commanditaires et protecteurs, mais aussi de tous les clients potentiels de ses cartes. Il faut se distinguer, souligner la supériorité de ses œuvres sur le gros des productions existantes. Comme l'explique Verdier, d'Anville y parvient en intervenant dans le Mercure de France de trois manières : en participant aux grandes disputes géographiques, comme le débat sur la forme de la terre, lequel fut à l'origine des expéditions en Laponie et en Équateur vers 1735 ; en répondant par des lettres ouvertes aux critiques qui lui sont adressées; en restituant sous la forme de dissertations abrégées, les raisonnements à l'origine des choix opérés dans ses cartes.

Une fois devenu académicien en France, d'Anville cesse d'écrire dans les journaux, ce qui semble corroborer l'hypothèse selon laquelle ceux-là constituaient avant tout une stratégie de communication. Sa nomination à l'Académie royale des inscriptions en 1754 scelle sa reconnaissance institutionnelle, tout en lui offrant un organe de diffusion de ses œuvres : les mémoires de l'académie. 
D'Anville ne se contente pas de se faire connaître par les journaux et par son travail. Il soigne son image de marque, une autre manière de créer et d'asseoir une réputation. L'élaboration de son ethos de savant constitue une stratégie de crédibilité. Vis-à-vis du public ou même d'autres savants, il se construit une image de solitude, d'austérité et de rigueur qui correspond alors à ce qu'on attendait d'un savant, et consolide son statut d'autorité en matière géographique. Il n'est pas inintéressant de noter qu'il employait, dans ses jeunes années une toute autre rhétorique. Évoquant ainsi la discussion à l'origine du texte publié dans le Mercure à 19 ans, il affirme que la mise par écrit fut difficile, mais, que " [son] amour propre se sentit piqué de ce désir ; il l'emporta sur [sa] paresse et sur quelques autres raisons ». Il ajoute plus loin : «c'est un vrai suplice [sic] pour un homme qui n'habite pas un cabinet volontiers" (d'Anville, 1717, 70-71). Le jeune homme se présente ainsi comme un inactif qui a eu bien du mal à se mettre au travail. À chaque âge sa rhétorique : il n'est pas bon, trop jeune, de jouer la carte du rat de bibliothèque.

À une époque où les carrières ne se font plus simplement à la cour, et se partagent entre les institutions et la faveur publique, la réputation acquiert une importance accrue. D'Anville, loin de l'image de savant un peu rétrograde que certains ont eu de lui (Broc, 1975, 35), a très vite saisi les enjeux de son temps et a su mettre en œuvre les stratégies nécessaires pour s'attirer la faveur de mécènes, s'attacher la reconnaissance de ses pairs, faire de son nom une marque de fabrique.

\section{III. À qui profite la gloire posthume de d'Anville?}

Après sa mort, loin de s'estomper, la réputation de d'Anville ne fait que croître. Une fois le principal bénéficiaire de cette réputation disparu, qu'est-ce qui motive biographes et admirateurs à multiplier les hommages, quand même l'intérêt commercial n'est plus en jeu? À l'exception des cartes du monde antique, et de quelques pays encore difficilement accessibles, la plupart des cartes de d'Anville sont périmées au bout de quelques dizaines d'années. Or, tout au long du XIX siècle, la gloire du géographe ne fait qu'augmenter.

Parce que la gloire ou la célébrité n'ont jamais rien d'une évidence et ne sont pas la simple conséquence naturelle du mérite, ces hommages rendus à d'Anville ne l'ont pas été sans raison. De fait, un savant brillant, une œuvre majeure, un acte héroïque n'ont jamais garanti l'accès à la postérité. Chaque époque construit son panthéon, conforme à ses valeurs et à ses ambitions (Bonnet, 1986, 217-223 ; Bonnet, 1998 ; Gérard, 1998, 31-48; Noiray, 1998, 143-158). Certes, les travaux de d'Anville, bien qu'en partie obsolètes, sont au XIX ${ }^{\mathrm{e}}$ siècle largement utilisés par les géographes, les explorateurs, les archéologues et les linguistes. Ses cartes sont ainsi la principale source de la première œuvre du jeune Jean-François Champollion, l'Égypte des pharaons, qui reprend telle quelle la carte de Haute Égypte (Champollion, 1811, 384-385). Cependant, cela n'explique guère pourquoi l'homme est célébré et statufié, son profil frappé sur des médailles.

47 Pour le comprendre, voici deux cas qui montrent que la célébration d'un savant dépasse les enjeux de sa propre postérité, et peut servir des desseins très différents selon le contexte, en l'occurrence la restauration du prestige national pour la Galerie métallique des grands hommes et la défense de la géographie érudite comme discipline pour les textes de Conrad Malte-Brun et Vivien de Saint-Martin. 
Le profil de d'Anville est frappé dans la Galerie métallique des grands hommes. Or il est assez rare, on l'a vu, qu'un géographe soit qualifié de "Grand homme ». Il ne paraît donc pas inutile de s'interroger sur la présence de d'Anville dans cet Olympe laïc. Le projet est conçu vers 1815 par Louis Bérard (1783-1859), politicien, industriel et haut fonctionnaire français. À l'époque, le projet de Panthéon accueillant les dépouilles de citoyens d'exception peine à se stabiliser. La définition du "grand homme " pose problème : en période d'instabilité politique, les héros des uns sont les ennemis des autres. Pourtant, en plein défaite, suite à la chute de Napoléon à Waterloo, il est plus que nécessaire de redonner aux Français vaincus confiance et fierté en leur pays. Le Mercure de France ne présente pas autrement le projet :

La Galerie métallique des grands hommes français est destinée à conserver, par le moyen des médailles, les traits de tous les littérateurs savans ou artistes célèbres qui ont concouru à la gloire de la France, et à la faire passer à la postérité la plus reculée. Cette entreprise éminemment nationale, et dont on n'a besoin que d'indiquer le but pour en faire l'éloge, a déjà été couronnée du plus heureux succès (Anonyme, 1817, 226-227).

Voilà donc à quoi sert alors un "grand homme": à raffermir un sentiment patriotique. Le choix de d'Anville n'a ici rien à voir avec un quelconque discours de légitimation géographique et savante.

En période de perpétuels revirements, le projet se veut prudemment apolitique. Il faut dire que Bérard est plutôt libéral et que la France est en pleine restauration monarchique. Les hommes de guerre et les princes qui incarnaient jusqu'alors la gloire sont écartés au profit d'hommes de sciences, de lettres et d'artistes, choisis par des figures de la classe dirigeante. ${ }^{12}$ Les sélectionnés sont majoritairement des hommes, même si on y trouve quelques femmes (Héloïse, Jeanne d'Arc, Marie de Sévigné, Anne de Staël). Les personnalités du XVIII ${ }^{e}$ siècle sont privilégiées (53 sur 119). Or d'Anville est une figure relativement consensuelle. Il est bon catholique, proche des princes et des rois, ce qui ne saurait nuire en pleine Restauration, sans être associé à la Contrerévolution ou à un quelconque parti, étant mort sept ans avant la Révolution (Leniaud, 2002, 197, n³). Deux géographes sont retenus pour apparaître dans cette Galerie métallique : d'Anville et Jean-Dominique Cassini (dit Cassini ${ }^{\text {er }}$ ). Les Sanson, les Delisle ou les Robert de Vaugondy ont été écartés. La renommée de d'Anville et de son œuvre était peut-être plus vivace, renforcée par la longévité de sa carrière. Certaines des personnalités choisies pour la Galerie firent débat, leur apport paraissant trop maigre (Xivrey, 1837, 349). Mais d'Anville semble avoir fait consensus. Ici, c'est donc la renommée du géographe qui vient servir le patriotisme et raviver l'honneur national.

51 Toutefois, le patriotisme qui sous-tend la notion de "grand homme » n'est pas toujours une fin en soi. Il peut à son tour servir de levier afin de défendre une géographie savante en difficulté institutionnelle et globalement victime d'une image dégradée auprès du public. Dans les deux textes qui vont être évoqués, la figure de d'Anville comme "grand homme » est construite pour être mise au service de la géographie. Le premier est de Conrad Malte-Brun, Sur l'édition complète des ouvrages de M. d'Anville, publié en 1810 dans les Annales des voyages. Il y déplore que le second volume des Ouvrages de M. d'Anville n'ait pu voir le jour, faute de souscripteurs. Le second, publié 45 ans plus tard, est intitulé de l'État de la cartographie en Europe, en 1855. Il s'agit de la retranscription d'un discours de Vivien de Saint-Martin prononcé la même année devant la Société de géographie, à l'occasion de l'Exposition universelle de Paris, la première organisée dans la capitale française. Bien qu'ils aient été écrits à près d'un 
demi-siècle de distance, les deux textes font part d'un même constat: la géographie savante est en difficulté, et fait face à de nombreuses critiques.

En 1810, Conrad Malte-Brun met l'accent sur la mauvaise image dont souffre la géographie auprès du public, pour qui elle n'est qu'enfantillages :

Que faire, au contraire avec ces gens qui ne voient dans toutes les sciences que la partie scolastique, qui [...] s'imaginent que la géographie n'est qu'une étude d'enfant, parce qu'en effet eux-mêmes ne savent pas plus de géographie qu'ils n'en ont appris dans leurs classes (Malte-Brun, 1810, 361).

La géographie se résume aux yeux du plus grand nombre à ce qui a été enseigné au collège À l'âge adulte, ce n'est guère plus qu'un divertissement de société :

Il ne s'agit point d'enseigner aux petits enfans où étoit situé Babylone, et qu'elles [sic] étoient les frontières de la Gaule ; il n'est pas non plus question d'amuser les grands enfans (Malte-Brun, 1810, 362).

La réputation de la géographie, et tout particulièrement de la géographie érudite, n'est pas meilleure auprès d'un public averti. Au contraire, selon Conrad Malte-Brun, ce public est largement responsable de son image déplorable. Les historiens la dénigrent comme trop peu littéraire. Elle est insuffisamment positive pour les scientifiques. Elle est même critiquée par certains cartographes spécialisés dans la géographie mathématique, que Malte-Brun qualifie de "dessinateurs de cartes », et qui cherchent à débarrasser la géographie de tout caractère transdisciplinaire :

Il y a des savans, il y a des ignorans également zélés pour décrier cette branche des connoissances humaines. Selon quelques dessinateurs de cartes, il ne devroit être question en géographie que des longitudes et latitudes; tout ce qui, dans le tableau moral et historique, intéresse le sentiment et élève l'ame, n'est à leurs yeux qu'une parure étrangère par laquelle des charlatans usurpent cette faveur publique, qui devroit être réservée exclusivement à des dissertations sur la projection stéréographique, conique, cylindrique et autres. Les gens de lettres vont à l'autre extrême : ils ne veulent admettre dans la géographie que les parties historiques, les détails susceptibles d'être traités en style élégant et fleuri. C'est ainsi que chaque classe de savans a ses prejugés à part sur la géographie qui, étant une science mixte, offre naturellement beaucoup de parties dans lesquelles les hommes, étrangers à l'ensemble de la science, se croient des juges compétens (Malte-Brun, 1810, 361).

La nature hétérogène de cette discipline « mixte » en fait une cible facile : comme le fait remarquer Malte-Brun, chacun se croit apte à en juger.

En 1855, la situation ne s'est pas améliorée, en témoigne le discours de Vivien de SaintMartin. Les préjugés du public ne font plus l'objet de commentaires, comme s'ils avaient perdu en importance. En revanche, la rivalité intestine entre les différentes branches de la géographie, que Malte-Brun dénonçait déjà, préoccupe Vivien de SaintMartin. À cause de la mauvaise réputation de la discipline, les géographes mathématiques font le choix de rompre avec leurs pairs. Ils cherchent à regagner une légitimité en se distinguant de la géographie historique et savante, qu'ils critiquent publiquement. L'expression de ce mépris se cristallise autour des œuvres de d'Anville, qui incarne le géographe érudit par excellence. Les critiques de son système de représentation de l'orographie sont une des expressions de ce " dédain »:

On a manifesté, dans nos nouvelles écoles cartographiques, un grand dédain pour le système suivi par d'Anville pour exprimer les montagnes; ces imperceptibles accents circonflexes, accompagnés de très légers traits d'ombre, ont, à ce qu'il parait, été trouvés fort ridicules. [...] Ces signes ne sont qu'une convention ; soit ; mais que sont donc les vôtres? (Vivien de Saint-Martin, 1855, 20). 


\section{désolidariser d'un géographe qui n'avait pas acquis une vraie légitimité à l'Académie} royale des sciences, ce dont témoigne l'éloge posthume signé par Condorcet. Selon lui, « il savait très peu de géométrie, et moins encore d'astronomie » et a obtenu sa place d'adjoint-géographe «quoique M. d'Anville eût traité la géographie plus en érudit qu'en astronome ou en géomètre » (Condorcet, 1785, 75).

Le reproche adressé à d'Anville de ne rien connaître aux mathématiques et à l'astronomie est un peu exagéré. On sait que ces matières étaient fort bien enseignées au Collège des Quatre nations où il étudia (Franklin, 1862). Il les transmet aussi au cours de sa carrière..$^{13} \mathrm{Il}$ n'en reste pas moins vrai qu'il les utilise très peu dans le quotidien de son étude (Haguet, 2010, 88-101). Ce n'est sans doute pas un grand expert en ces matières. Lui-même le reconnait volontiers :

M. de Fontenelle [...], me fit un jour l'honneur de me dire qu'il étoit supris [sic] de me voir sans sollicitude à me frayer l'entrée de l'Académie royale des sciences. Je répondis à cela, que sur un sujet de cette conséquence il y avoit plus de sagesse dans la retenue que dans une confiance téméraire. [...]. J'ajouterai à cela, que des sentiers qui conduisent modestement à ce qu'on appelle érudition, m'étoient plus familiers que les routes qui, selon l'estime ordinaire, font arriver à des connoissances auxquelles on donne par préférence le nom d'exactes en toute rigueur. ${ }^{14}$

Enfin, comme l'a montré l'historienne Hélène Blais, la fragilité institutionnelle de la géographie s'aggrave encore au XIX ${ }^{e}$ siècle, avec la dissolution des académies et la fondation de l'Institut. Dans un premier temps, la géographie est placée avec les sciences morales et politique. Mais en 1803, tandis que cette classe des sciences morales est supprimée, la géographie passe aux mathématiques, mais seulement pour la moitié des effectifs. Les autres membres, dont la pratique est plus érudite que mathématique, sont répartis dans la section histoire et littérature, sans être identifiés sous l'étiquette "géographie». Autrement dit, la géographie érudite devient invisible dans les institutions, et disparaît des textes officiels (Blais, 2006, 95-112). Par ailleurs, elle est de plus en plus critiquée par son absence d'ambition théorique, comme l'a montré Anne Godlewska, en révélant les critiques dont fait l'objet l'enseignement d'Edme Mentelle, géographe de cabinet et professeur à l'École normale vers 1794 (Godlewska, 1999).

Ce n'est donc pas sans raison que Vivien de Saint-Martin déplore qu'elle ne bénéficie d'aucun soutien financier institutionnel, pourtant indispensable à sa pratique :

La carrière géographique, si l'on en sépare toute préoccupation commerciale, n'est pas de celles qui peuvent conduire à un grand dédommagement pécuniaire. La réunion des matériaux est trop coûteuse, l'élaboration trop longue et le produit trop faible. Je ne voudrais pas comparer la position particulière que la sollicitude de l'Académie et la munificence d'un prince ami des lettres avaient faites à d'Anville, avec la condition actuelle de l'homme de lettres et du savant livré à ses seules ressources; mais il est bien évident que la réunion des conditions nécessaires à l'édification d'une grande œuvre géographique devient chaque jour plus difficile (Vivien de Saint-Martin, 1855, 257).

Cependant, la géographie d'érudition a encore un rôle central à tenir au XIX $\mathrm{X}^{\mathrm{e}}$ siècle. Le travail de compilation demeure indispensable pour composer une image cohérente du monde à partir d'un ensemble hétérogène de récits et de mesures prises sur le terrain. Quoique certains cherchent à mettre les érudits à distance de la géographie, ils restent nécessaires à son élaboration, même en ce XIX ${ }^{e}$ siècle positif. Sans eux, point de cartes générales possibles, et c'est parce qu'il en a bien conscience que Vivien de Saint-Martin s'attache à défendre la cause de la géographie de cabinet auprès des membres de la 
Société de géographie. C'est donc là qu'intervient l'invention d'un passé glorieux de la géographie érudite, incarné par d'Anville, "grand homme " quasi érigé en fondateur de la géographie moderne.

L'éclat de la réputation de d'Anville sert à mieux faire sentir la déchéance de la géographie savante, et avec elle, celle d'un certain prestige de la France. Là encore, l'argumentation est symétrique, chez Malte-Brun et Vivien de Saint-Martin. Chacun d'eux fait vibrer la corde de la sensibilité nationale afin de rallier leurs auditeurs et lecteurs à leur cause.

Chez Malte-Brun, l'argumentation est la suivante. La gloire de d'Anville en France est manifeste. Elle n'est pourtant pas accompagnée d'un véritable intérêt pour le travail du géographe, puisqu'elle n'aboutit à aucune action concrète en faveur de son héritage. Dans ce passage, qu'il affirme extrait d'une lettre reçue d'un Anglais, est fustigée la superficialité du sentiment national français :

Le peu de zèle qu'on montre en France pour les CEuvres complètes de d'Anville semble assez bien marquer la différence qui existe entre la vanité nationale des Français et l'orgueil patriotique des Anglais. Qu'on parle géographie devant un Français, absolument étranger à cette science, il vous répétera bien haut le nom de l'immortel d'Anville; il vous donnera à entendre que ce grand homme a épuisé toutes les mines de la géographie, et n'a rien laissé à faire à ceux qui sont venus après lui. [...] C'est sur ce ton que parlent vingt journalistes de Paris, très-persuadés, sans doute, qu'ils défendent la gloire de leur pays et de leur nation (Malte-Brun, 1810, 357)..$^{15}$

Martin mesure la décadence de la géographie érudite. Et c'est également en aiguillonnant l'orgueil patriotique de ses compatriotes, que Vivien de Saint-Martin espère créer un sursaut en faveur de celle-ci. La situation ne s'est pas arrangée, bien au contraire. Car à la vanité paresseuse et aux critiques ont fait place une « couche épaisse d'indifférence et d'inertie [...], plus fatale qu'une opposition » (Vivien de Saint-Martin, 1855, 28).

Tout d'abord, Vivien de Saint-Martin brosse le portrait d'une géographie française au passé prestigieux, dominée par des Sanson, des Cassini, de sorte qu' « une des gloires de la France, nous pouvons du moins le rappeler avec orgueil, est d'avoir été, avant aucun autre pays de l'Europe, le siège des grandes études géographiques » (Vivien de SaintMartin, 1855, 11). D'Anville incarne l'apogée de cet âge d'or de la cartographie française, et à ce titre, son cas est développé sur plusieurs pages : «Quelque importante qu'eût été la réforme de Delisle, ce n'était cependant qu'un premier pas ; d'Anville, un demi-siècle plus tard, allait accomplir un progrès plus sensible encore et plus complet " (Vivien de Saint-Martin, 1855, 12). L'orateur prend bien soin de situer cette prééminence dans un contexte où les cartes de d'Anville sont notamment supérieures aux cartes anglaises et allemandes (Vivien de Saint-Martin, 1855, 14).

Ce long rappel du règne du géographe parisien sur la cartographie européenne a bien sûr pour dessein de rendre le portrait du déclin de la géographie française plus douloureux pour l'auditoire :

Les cartes exécutées chez nous dans les dernières années du XVIII ${ }^{\mathrm{e}}$ siècle et au commencement du siècle actuel témoignent assez de cette rapide décadence. Pour 
nous relever de cette décadence, il aurait fallu un autre d'Anville (Vivien de Saint-

Martin, 1855, 14). Vivien de Saint-Martin n'a pas une conception essentialiste du savant d'exception. Pour lui, celui-ci ne peut exister qu'à partir d'un terreau favorable, notamment au niveau institutionnel.

69 À cette fin, il développe le cas de Pierre Lapie (1779-1850), premier géographe du roi, qui aurait eu l'étoffe $d^{\prime}$ ' "un autre d'Anville », s'il avait reçu le soutien financier indispensable :

M. Lapie réunissait incontestablement une grande partie des qualités nécessaires au géographe savant. Il les aurait eues toutes, je le crois, si le côté commercial de ses travaux ne l'eût poussé à une production multipliée, au milieu de laquelle il est impossible de réserver à la pensée le temps nécessaire pour la maturité des recherches, aussi bien que pour la critique des matériaux et la lente élaboration des éléments accumulés (Vivien de Saint-Martin, 1855, 15).

70

Faute d'héritage, de rentes, de dot, et faute surtout de soutien financier des institutions savantes, Lapie doit gagner sa vie. Et pour le faire tout en restant cartographe, il est contraint, selon Vivien de Saint-Martin, de faire de son art un commerce, le second réussissant au dépend du premier. En matière de cartographie érudite, on serait tenté de renverser l'adage : "L'argent, c'est du temps ».

71 La société bourgeoise et industrielle du XIX siècle n'est pas la société de l'Ancien Régime. Elle attend un retour sur investissement tangible à ses financements. Pragmatique, elle s'intéresse peu aux progrès de la géographie érudite et historique :

Et puis, faut-il le dire, l'indifférence générale pour les études purement spéculatives y apporte un obstacle de plus. On a de bonnes cartes marines pour les besoins de la navigation: pour ceux de la guerre et de l'industrie, on a les magnifiques cartes officielles : que faut-il de plus ? [...] Tel est le raisonnement de la masse, et l'on ne peut nier qu'au point de vue strictement utilitaire il ne soit concluant (Vivien de Saint-Martin, 1855, 22).

«Utilitaire » : le mot est lâché. Ce qui est "utile» doit être compris comme ce qui rapporte de l'argent, directement ou indirectement. C'est bien ainsi que Vivien de Saint-Martin l'entend, quand il demande « de quelle utilité pratique sera cette œuvre de géographie savante pour les besoins journaliers de la vie et le développement de la fortune publique. » (Vivien de Saint-Martin, 1855, 22) »

73 Toute l'habileté rhétorique de l'orateur va consister à introduire dans un premier temps le concept de prestige national, afin de tenter de faire valoir que la gloire d'un pays ne se mesure pas qu'à son développement technologique et à sa puissance économique :

Vous croyez, et je crois comme vous, Messieurs, que la grandeur d'une nation et d'un siècle, et leur place définitive dans l'histoire du monde, s'appuient sur autre chose que le développement industriel (Vivien de Saint-Martin, 1855, 22).

Vivien de Saint-Martin pose ensuite une équivalence entre géographie érudite et gloire nationale, pour mieux asséner son coup de grâce. La France n'a plus de grande géographie d'érudition, c'est donc qu'elle n'est plus qu'une puissance de second plan. Après avoir heurté le sentiment patriotique de son auditoire, il ne lui reste plus qu'à suggérer la solution :

Maintenant, Messieurs, vis-à-vis de ce tableau presque décourageant et malheureusement trop vrai, une question se présente naturellement; on se 
demande par quelles causes l'Angleterre et l'Allemagne du Nord, placées à ce qu'il semble dans des conditions de vie intellectuelle semblables aux nôtres, ont marché en sens inverse dans les voies de la cartographie critique ? Comment, de l'état pour le moins médiocre où elles étaient l'une et l'autre sous ce rapport il y a un quart de siècle à peine, elles sont montées au premier rang [...] (Vivien de Saint-Martin, 1855, 23).

Bien sûr, la réponse est dans la création ou le soutien aux institutions savantes. Le dynamisme de la Société royale de géographie pour l'Angleterre et le soutien des universités allemande aux savants ont permis à la " géographie critique » de connaître un développement considérable tandis qu'elle connaissait en France un inexorable déclin.

76 Là encore, comme chez Malte-Brun, quelques décennies plus tôt, la comparaison avec les voisins anglais et allemands est employée comme aiguillon pour faire réagir les Français: c'est dire à quel point les géographes peinent à faire entendre leurs avertissements.

Parce qu'il est ambigu, entaché de superficialité, le rôle de la célébrité dans une carrière, mais aussi dans l'élaboration même d'une œuvre, n'est pas souvent mis en avant dans l'analyse des parcours scientifiques. Pourtant, la célébrité contribue fortement à densifier les réseaux de sociabilité et les échanges d'information, à augmenter les moyens financiers, sans compter qu'elle constitue très certainement un encouragement psychologique pour celui qui s'investit corps et âme dans son domaine. En géographie, elle favorise également la réussite commerciale puisqu'elle permet d'écouler davantage de cartes, la marque "d'Anville", si on ose dire, étant gage de qualité. La diffusion, voire la création d'une célébrité posthume du savant diffère de sa réputation de son vivant en ce qu'elle ne remplit pas les mêmes objectifs. Elle ne sert plus à servir un homme et son travail, mais une discipline tout entière, négligée par l'État. En d'autres termes, l'exemple de d'Anville montre que la célébrité peut aussi être un discours, un peu forcé, au service d'une pratique savante et d'une discipline.

Témoin de l'ambiguïé de la position de d'Anville au panthéon des grands hommes, le portrait de d'Anville a bien été choisi pour apparaître dans Les illustres français de Nicolas Ponce (1816) qui contient plusieurs portraits en médaillons superposés sur une même planche, les uns cachant parfois entièrement les autres, laissant leur seul nom apparent. De manière significative, le portrait de d'Anville est recouvert par le médaillon d'un autre cartographe : Jean-Dominique Cassini.

\section{BIBLIOGRAPHIE}

Anonyme, 1817, « Galerie métallique des grands hommes français », Mercure de France, août, p. 226-227.

Anville J.-B. d' (attribué à), 1717, « Description de l'isle de Ceylan », Mercure de France, novembre, p. $70-130$. 
Anville J.-B. d', 1719, « Lettre de l'auteur de la carte d'Aragon annoncée dans le dernier Mercure ", Le Nouveau Mercure, novembre, p. 74-82.

Anville J.-B. d', 1766, Mémoires sur l'Égypte ancienne et moderne, Paris, Imprimerie royale, 277 p.

Anville J.-B. d', 1777, Considérations générales sur l'étude et les connoissances que demande la composition des ouvrages de géographie, Paris, Imprimerie de Lambert, 116 p.

Agulhon M., 1998, « Nouveaux propos sur les statues de "grands hommes" au XIX ${ }^{\mathrm{e}}$ siècle », Romantisme, Les Grands hommes, vol. 28, n 100, p. 11-16.

Allard S., Scherf G., 2006, Portraits publics, portraits privés, 1770-1830, exposition Galeries nationales du Grand Palais, Paris, 4 octobre 2006-9 janvier 2007, Paris, Réunion des musées nationaux, 383 p.

Amalvi Ch., 1998, «L'exemple des grands hommes de l'histoire de France à l'école et au foyer (1814-1914)», Romantisme, Les Grands hommes, vol. 28, n 100, p. 91-103.

Balzac H. de, 1891, La Cousine Bette, Paris, C. Levy, 1891, 569 p.

Bibliothèque nationale, 1855-1895, Catalogue de l'histoire de France, Paris, Firmin Didot, 18 vol.

Blais H., 2006, « La géographie académique entre sciences et belles-lettres (autour de la scission de 1803) ", Blais H., Laboulais I., Géographies plurielles. Les sciences géographiques au moment de l'émergence des sciences humaines (1750-1850), Paris, L'Harmattan, p. 95-112.

Bonnet J. C., 1986, « Les morts illustres, raison funèbre, éloge académique, nécrologie », Pierre Nora éd., Les Lieux de mémoire, Paris, Gallimard, vol. 3, p. 217-223.

Bonnet J. C., 1998, Naissance du Panthéon, Essai sur le culte des grands hommes, Paris, Fayard, 414 p.

Bonnet J. C., 2001, « Le Culte des grands hommes en France au XVIII ${ }^{\mathrm{e}}$ siècle ou la défaite de la monarchie », Modern Language notes (MLN), volume 116, number 4, September 2001 (French Issue), p. 689-704.

Bougainville L. A. de, 1772, Voyage autour du monde par la frégate la Boudeuse et la flûte l'Étoile, Paris, chez Saillant et Nyon, 3 vol. in- $8^{\circ}$

Broc N., 1975, La Géographie des philosophes, géographes et voyageurs français au XVIII ${ }^{e}$ siècle, Paris, Ophrys, 595 p.

Bruno G. [Fouillée A.], Belin E., 1878, Tour de la France par deux enfants, Paris, Belin, 312 p.

Castel L. B., 1737, « Dissertation sur la célèbre terre de Kamschatka et sur celle d'Yeço », Mémoires de Trévoux (juillet 1737)», p.1156-1226.

Champollion J. F., 1811, L'Égypte des Pharaons, Grenoble, Peyronard, 67 p.

Choiseul-Gouffier M. G. F. A. de, 1822, Voyage pittoresque de la Grèce, Paris, J.-J. Blaise, vol. III, p. 17.

Condorcet M. J. A. N. de Caritat de, 1785, « Éloge de d'Anville », Histoire de l'Académie royale des Sciences, année M DCC LXXXII, Paris, Imprimerie nationale, 1785, p. 69-77.

Dacier B.-J., 1802, «Éloge de M. d'Anville », Notice des ouvrages de M. d'Anville, Paris, Fuchs et Demanne, p. 17-44.

Dewachter M., 1994, « Les Voyageurs français et la Nubie », dans Gratien B., Le Saout F., Nubie, les cultures antiques du Soudan, Lille, Université de Charles de Gaulle, p. 35-43.

Franklin A., 1862, Recherches historiques sur le collège des Quatre-Nations, Paris, Auguste Aubry, 205 p. 
Furtado J., 2012, Oráculos da Geografia Iluminista, Dom Luis da Cunha e Jean-Baptiste Bourguignon d'Anville na construção da cartogafía do Brasil, Belo Horizonte, Universidade Federal de Minas Gerais, 707 p.

Gérard A., 1998, « Le Grand homme et ma conception de l'histoire au XIX siècle », Romantisme, le Grand Homme, 100, p. 31-48.

Godlewska A., 1999, Geography unbound, French geographic science from Cassini to Humboldt, Chicago, London, The University of Chicago press.

Gourdon de Genouillac H., 1879, Paris à travers les siècles, Paris, F. Roy, 6 vol.

Haguet L., 2010, « J. B. d'Anville as armchair mapmaker, the impact of production contexts on his work », Imago Mundi, vol. 63, part 1, p. 88-101.

Haguet L., 2017, « Splendeur et décadence d'un 'grand homme' : réception et postérité de d'Anville et son œuvre ", dans Haguet L., Hofmann C., Une carrière de géographe au siècle des Lumières, Oxford, Oxford University Press, p. 311-360.

Haguet L., Hofmann C., 2017, Une carrière de géographe au siècle des Lumières, Oxford, Oxford University Press, $544 \mathrm{p}$.

Hillairet J., 1997, Dictionnaire historique des rues de Paris, Paris, Éditions de Minuit, 2 vol., 739 p. et $740 \mathrm{p}$.

Le Normand-Romain A. (dir.), 2007, Rodin et le Bronze, Paris, Réunion des musées nationaux, Musée Rodin, 2007, 2 vol., 822 p.

Lentz T., 2013, Napoléon, Paris, La Boétie, 214 p.

Leniaud J.-M dir., Procès-verbaux de l'Académie des Beaux-arts, 1816-1820, t. 2, Paris, École des Chartres, 2002, $600 \mathrm{p}$.

Lilti A., 2014, Figures publiques. L'invention de la célébrité (1750-1850), Paris, Fayard, 430 p.

Malte-Brun C., 1810, "Sur l'édition complète des Ouvrages de M. d'Anville », Annales des voyages de la géographie et de l'histoire, Paris, Buisson, t. 10, p. 356-363.

Mandroux-França M.-T., 1986, « La collection d'estampes du Roi Jean V de Portugal, une relecture des notes manuscrites de Pierre-Jean Mariette ", Revue de l'art, 73, p. 49-54

Manne L. Ch. J. de, 1834, Cuuvres de d'Anville publiées par M. de Manne, Paris, Levrault, vol. 1, 677 p.

Marcel G., 1904, «Lettres inédites du cardinal Domenico Passionei à d'Anville », Bulletin de géographie historique et descriptive, $\mathrm{n}^{\circ} 3,44 \mathrm{p}$. (tiré à part)

Mormiche P., 2010, « Participer à l'éducation des princes : D’Anville et son élève Louis XV (1718-1730) », dans Haguet L., Hofmann C., Une carrière de géographe au siècle des Lumières, Oxford, Oxford University Press, p. 23-51.

Noiray J., 1998, « Figures du savant », Romantisme, le Grand Homme, 100, p. 143-158.

Ponce N., 1816, Les Illustres Français, ou Tableaux historiques des grands hommes de la France, pris dans tous les genres de célébrité, Paris, Ponce.

Quatremère de Quincy Antoine Chrysostôme, 1834, « Notice historique sur la vie et les ouvrages de M. Duvivier ", Recueil de notices historiques lues dans les séances publiques de l'Académie royale des beaux-arts, Paris, Adrien Le Clere, p. 176-191.

Robert de Vaugondy D.,1755, Essai sur l'histoire de la géographie, Paris, Boudet, 422 p. 
Verdier N., 2017, « Entre publicité, débat scientifique et vulgarisation : Jean Baptiste d'Anville dans les journaux », dans Haguet L., Hofmann C., Une carrière de géographe au siècle des Lumières, Oxford, Oxford University Press, p. 237-259.

Veyrat G., 1892, Les Statues de l'hôtel de ville, Paris, Librairies-imprimeries réunies, 352 p.

Vivien de Saint-Martin L., 1855, De l'État de la cartographie en Europe, et particulièrement en France : à propos de l'Exposition universelle, Paris, Imprimerie impériale, $23 \mathrm{p}$.

Xivrey J.-B. de (1837), Essais d'appréciations historiques, ou examen de quelques points de philologie, de géographie, d'archéologie et d'histoire, Paris, Desforges, 2 vol., 282 et 362 p.

Sitographie

Trésor de la langue française informatisé. http://stella.atilf.fr/Dendien/scripts/tlfiv5/advanced.exe? $8 ; \mathrm{s}=50164875$. Consulté le 15 janvier 2020

Jean-Baptiste d'Anville, Un cabinet savant à l'époque des Lumières

https://danville.hypotheses.org/

Consulté le 15 janvier 2020

\section{NOTES}

1. D'Anville assure avoir eu un premier goût pour le dessin dans son enfance, avant de se passionner pour la géographie. Alençon, Archives départementales de l'Orne, SHAO (252J224), « Projet de don de la collection », f.1.

2. Voir le Trésor de la langue française informatisé.

3. Paris, BnF, Département des manuscrits, NAF 22147, "Contrat et papiers relatifs au contrat passé entre Jean-Baptiste d'Anville et les libraires Desaint, Saillant et Durand (Paris, 24 novembre 1759)».

4. Alençon, Archives départementales de l'Orne, Collection de la société historique et archéologique de l'Orne, Cote 252J 224, « Mémoire sur un projet de géographie »

5. Musée national des châteaux de Versailles et de Trianon, MV 4414 ; INV 9000 ; MN 11. Il fut acquis par l'État le 11 janvier 1849. L'attribution du tableau à Louis Tocqué provient d'une copie incomplète du catalogue de vente conservé au Rijksbureau voor Kunsthistorische Documentatie, La Haye (RKDH).

6. Ernest Mouchez, Plan d'atterrage du golfe d'Anville (côtes de Tartarie), août 1852. BnF, Département des cartes et plans, Ge BB 3, vol. 67, carte 1467, planche 11 (carton d'une carte principale).

7. Le portrait par Louis Tocqué ne semble pas avoir été reproduit.

8. Voir par exemple le portrait de d'Anville, dessiné et gravé par Lecerf d'après Pierre Simon Benjamin Duvivier, qui ne ressemble plus que de très loin à l'original, publié dans Conrad MalteBrun, Annales des voyages de la géographie et de l'histoire (Paris, Buisson, 1810), t.X, après le p. 357. Bibliothèque nationale de France, PHS, G 18346 (après p. 357).

9. Voir par exemple, Benjamin Duvivier, Augustin de Saint-Aubin, Portrait de profil de Jean-Baptiste Sanson (1724-1798), prêtre, s. d. Versailles, châteaux de Versailles et de Trianon, Album Louis Philippe, Estampes, LP83.82.2.

10. Alençon, Archives départementales de l'Orne, Collection de la société historique et archéologique de l'Orne, cote 252J 224, « Manuscrits de d'Anville ».

11. Alençon, Archives départementales de l'Orne, SHAO (252J224), «Mémoire de M. d'Anville dans lequel, indépendamment de quelques articles qui sont personnels, il traite de plusieurs 
points très-intéressans pour le fond de la géographie », [p. 12]. Mis en ligne par Lucile Haguet. Site web: http://f.hypotheses.org/wp-content/blogs.dir/239/files/2011/11/m\%C3\%A9moireautobiographique.pdf

12. Le comité de sélection d'après le Mercure: Armand Emmanuel du Plessis, duc de Richelieu, ministre des affaires étrangères, Joseph Lainé, ministre de l'intérieur, Élie Decazes, ministre de la police, Jules Jean-Baptiste François de Chardebœuf, comte de Pradel, ministre au département de la Maison du roi, Jules Anglès préfet de police, Prosper Bruyière, Baron de Barante, directeur des contributions indirectes, Monsieur de Vinci, pair de France. La liste exhaustive n'est toutefois pas connue.

13. Sur l'enseignement dispensé par d'Anville, voir l' «Affiche du 8 octobre 1753 », AN, Marc C/ 7/6 dossier d'Anville. L'affiche est manquante depuis 2016.

14. Alençon, Archives départementales de l'Orne, SHAO (252J224), 'Mémoire de M. d'Anville dans lequel, indépendamment de quelques articles qui sont personnels', p.1-16.

15. Les italiques sont dans le texte original.

\section{RÉSUMÉS}

Il peut sembler provocateur d'interroger la carrière et l'influence d'un savant sous le prisme de la célébrité et de ses déclinaisons. La question est cependant féconde, en témoigne l'exemple du géographe des Lumières Jean-Baptiste d'Anville (1697-1782), savant austère, dont le parcours semble à mille lieues de l'éclat superficiel de la célébrité. Pourtant, pour un géographe du XVIII ${ }^{\mathrm{e}}$ siècle, la construction non seulement d'une réputation mais d'une célébrité a pu jouer un rôle majeur dans la pratique de son art (obtention d'information, soutien matériel, vente accrue de cartes). Post mortem, la célébrité de d'Anville est exploitée pour défendre la géographie d'érudition auprès des institutions (les académies, l'Institut). Le fait même que la célébrité du savant puisse être mise en doute (d'Anville était-il si célèbre que certains l'ont prétendu ?), son caractère éventuellement artificiel, montre à quel point la célébrité peut être un instrument construit sur mesure, y compris dans un contexte académique.

It may seem provocative to question the career and influence of a scientist through the lens of fame and its variations. The question is fertile, however, as shown by the example of the Enlightenment mapmaker Jean-Baptiste d'Anville (1697-1782), an austere scholar, whose carrier seems a thousand miles from the superficial brilliance of celebrity. However, for an 18th century mapmaker, building reputation and celebrity may have played a key role in the practice of his art (obtaining information, material support, increased sale of maps). Post-mortem, the fame of D'Anville is exploited to defend a geography made in an office by scholars before institutions (academies, Institut).The very fact that the celebrity of the scientist can be questioned (was d'Anville so famous that some claimed it?), its possibly artificial character, shows to what extent fame can be a tailor-made instrument, even in an academic context.

\section{INDEX}

Mots-clés : Géographie des Lumières, célébrité, histoire des sciences, histoire de la cartographie Keywords : Enlightenment geography, fame, History of sciences, History of mapmaking 


\section{AUTEUR}

\section{LUCILE HAGUET}

Docteur en histoire, conservatrice à la bibliothèque municipale classée du Havre

Adresse professionnelle : Lucile Haguet, Service patrimoine, Bibliothèque Armand Salacrou, 17 rue Jules Lecesne, 76600 Le Havre

Adresse personnelle : Lucile Haguet, 35 chaussée John Kennedy, 76600 Le Havre

Adresse électronique : lucile.haguet@lehavre.fr 